\title{
Expression of Extracellular Superoxide Dismutase Protein in Diabetes
}

\author{
Chul Han Kim \\ Department of Plastic and Reconstructive Surgery, Soonchunhyang University College of Medicine, Seoul, Korea
}

Background Diabetes is characterized by chronic hyperglycemia, which can increase reactive oxygen species (ROS) production by the mitochondrial electron transport chain. The formation of ROS induces oxidative stress and activates oxidative damage-inducing genes in cells. No research has been published on oxidative damage-related extracellular superoxide dismutase (EC-SOD) protein levels in human diabetic skin. We investigated the expression of EC-SOD in diabetic skin compared with normal skin tissue in vivo.

Methods The expression of EC-SOD protein was evaluated by western blotting in 6 diabetic skin tissue samples and 6 normal skin samples. Immunohistochemical staining was also carried out to confirm the EC-SOD expression level in the 6 diabetic skin tissue samples.

Results The western blotting showed significantly lower EC-SOD protein expression in the diabetic skin tissue than in the normal tissue. Immunohistochemical examination of EC-SOD protein expression supported the western blotting analysis.

Conclusions Diabetic skin tissues express a relatively small amount of EC-SOD protein and may not be protected against oxidative stress. We believe that EC-SOD is related to the altered metabolic state in diabetic skin, which elevates ROS production.

Keywords Skin / Diabetes mellitus / Superoxide dismutase
Correspondence: Chul Han Kim Department of Plastic and Reconstructive Surgery, Soonchunhyang University College of Medicine, 59 Daesagwan-ro, Yongsan-gu, Seoul 140-743, Korea Tel: +82-2-709-9283

Fax: +82-2-796-3543

E-mail:kchann@hanmail.net

This work was supported by the Soonchunhyang University Research Fund.

No potential conflict of interest relevant to this article was reported.

\section{INTRODUCTION}

Diabetes is a critical disease prevalent throughout the world's population. The damage it causes arises from a number of pathologic mechanisms including oxidative stress [1]. Oxidative stress is caused by a cascade of reactive oxygen species (ROS) released from the mitochondria, a process related to type 1 diabetes, which is induced by apoptosis of pancreatic beta-cells, and type 2 diabetes, which is induced by insulin resistance [2]. ROS and the resulting oxidative damage are closely correlated with the pathogenesis and complications of diabetes [3]. Physiologically balanced ROS levels are involved in cell signaling and protection from various pathogens. A dysregulated ROS concentration may contribute to the development of a wide range of human diseases, such as diabetes, cancer, hypertension, atherosclerosis, and premature aging [1]. The evidence indicates the crucial role of oxidative stress in diabetes-related tissue injury [4]. The increased insulin resistance and reduced insulin secretory response induce an impaired glucose tolerance, leading to oxidative stress by the production of higher levels of ROS [4]. ROS play an important role in the activation of stress responsive signaling pathways regulating the expression of genes responsible for cellular damage.

While cells have a wide range of defense mechanisms against free radicals, free radicals bypass defense systems and attack and modify subcellular components, including proteins, lipids, and 
nucleic acids [5]. Proteins are a prominent target of oxygen free radicals and other reactive species [5]. Superoxide dismutases (SOD) are the first and most important major line of antioxidant defense systems against ROS, particularly superoxide anion radicals [6]. Among the SOD isoenzymes, extracellular superoxide dismutase (EC-SOD) was most recently discovered and is the principal defense mechanism against superoxide-mediated damage to both the cell surface and the extracellular matrix proteins [7]. Kimura et al. [8] reported a positive correlation between serum EC-SOD levels and the severity of vascular complications in diabetes. However, little is known about the role of ECSOD in the diabetic skin in vivo.

The aim of this study was to investigate the expression of ECSOD in vivo. We hypothesized that EC-SOD protein expression will be lower in diabetic skin tissue than in normal skin tissue. We studied the expression of EC-SOD in normal skin tissues and diabetic skin tissues by using western blot and immunochemistry.

\section{MATERIALS ANID METHODS}

\section{Tissue samples}

The Institutional Review Board of a university hospital in Seoul, Korea reviewed and approved this research protocol involving the use of tissue samples. A total of 6 normal skin tissue samples and 6 diabetic skin samples were obtained from patients who underwent surgery between December 2012 and January 2013 in the Department of Plastic and Reconstructive Surgery at the same university hospital. Informed consent was obtained from the patients before surgery. The normal skin tissues were collected from the back of 6 women who had breast reconstruction using the latissimus dorsi flap procedure. Diabetic skin samples were obtained from patients undergoing amputation surgery. A portion of the specimens were frozen in liquid nitrogen immediately after resection for western blot analysis, and stored at $-80^{\circ} \mathrm{C}$. For the immunohistochemical studies, the stored formalin-fixed, paraffin-embedded samples, including the 6 diabetic skin tissues and 6 normal skin tissues, were used.

\section{Immunohistochemical staining}

The paraffin sections were deparaffinized in xylene, rehydrated in a $10 \mathrm{mM}$ citrate buffer ( $\mathrm{pH} 6.0$ ), and heated in a microwave oven for 15 minutes to restore the antigens. To suppress endogenous peroxidase within the tissues, the samples were treated with $3 \%$ peroxide for 5 minutes, then with a blocking solution for 30 minutes. Slides were incubated with the primary EC-SOD rabbit monoclonal antibody (1:250; Catalog number ab171738, Epitomics, Cambridge, MA, USA) in a humid chamber for 60 minutes. The tissue staining was visualized with 3,3 'diamino- benzidine (ScyTek, Logan, UT, USA) substrate chromogen solution.

\section{Western blot analysis}

The tissue samples were homogenized in WCE buffer (25 mM HEPES [pH 7.7], $0.3 \mathrm{M} \mathrm{NaCl}, 1.5 \mathrm{mM} \mathrm{MgCl}_{2}, 0.2 \mathrm{mM}$ ethylenediaminetetraacetic acid, $0.1 \%$ Triton X-100, $0.5 \mathrm{mM}$ dithiothreitol, $20 \mathrm{mM}$ glycerol phosphate, $0.1 \mathrm{mM} \mathrm{Na}_{3} \mathrm{VO}_{4}, 2 \mathrm{~g} /$ $\mathrm{mL}$ leupeptin, $2 \mathrm{~g} / \mathrm{mL}$ aprotinin, $1 \mathrm{mM}$ phenylmethylsulfonyl fluoride, and a protease inhibitor cocktail tablet [Boehringer Mannheim, Mannheim, Germany]). The tissue suspension was rotated at $4^{\circ} \mathrm{C}$ for 10 minutes. The supernatants were collected, stored at $-70^{\circ} \mathrm{C}$, and used for western blotting. Proteins from tissue were separated by SDS-PAGE using NuPAGE $4 \%$ to $12 \%$ bis-Tris gels (Invitrogen, Carlsbad, CA, USA, NP0335Box) and then transferred to Immobilon-P membrane. The membrane was blocked using $5 \%$ bovine serum albumin (BSA) in TBS$\mathrm{T}$ (20 mM Tris, pH 7.6, $130 \mathrm{mM} \mathrm{NaCl}$, and 0.1\% Tween 20) solution. It was reacted with the primary antibody diluted to a 1:1,000 concentration at $4^{\circ} \mathrm{C}$ for 16 hours. With a washing buffer and TBST buffer (10 mM Tris-Cl, pH 8.0, $150 \mathrm{mM} \mathrm{NaCl}$, $0.05 \%$ Tween 20 ) it was washed well 4 times, for 10 minutes, 10 minutes, 15 minutes, and 15 minutes and then reacted for 1 hour with anti-rabbit IgG (Cell Signaling technology, \#7074, Danvers, MA, USA)-horseradish peroxidase-linked speciesspecific whole antibody diluted to 1:10,000. After the reaction with the secondary antibody, it was washed well twice for 10 minutes each and then twice for 15 minutes each. Proteins on the membrane were detected using an enhanced chemiluminescence solution kit (Amersham, Bucks, UK). The membranes were stripped and reblotted with anti-actin antibody (Sigma, catalog number A5441, St Louis, MI, USA). Primary rabbit anti EC-SOD (Catalog number SOD-105) was purchased from Stress Gen Biotechnology Corp. (Victoria, BC, Canada).

\section{Assessment of western blot analysis}

The relative abundance of the protein expression of each sample was analyzed by Phosphor-Imager software (TINA, Raytest, Straubenhardt, Germany). The expression levels of diabetic skin and normal skin tissues were compared.

\section{Statistical analysis}

The data from the Raytest TINA software were analyzed using SPSS ver. 17.0 (SPSS Inc., Chicago, IL, USA) and statistical significance was set at a P-value less than 0.05. Data are presented as mean \pm standard deviation (SD). For the comparison of nonnormally distributed variables, we used the Mann-Whitney U test. 


\section{RESULTS}

\section{Immunohistochemical examination}

The immunohistochemical study showed that EC-SOD protein was strongly expressed in normal skin tissue. Strong immunohistochemical expression of EC-SOD was demonstrated in the entire epidermis and appendages of normal skin tissue. However, EC-SOD was found to be weakly expressed in diabetic skin (Fig. 1).

\section{Western blot analysis}

The results of western blot analysis showed that EC-SOD was less expressed in diabetic skin tissues compared with normal human skin tissues (Fig. 2A). The amount of expression in western blotting shown depicts less expression in diabetic skin tissues compared with normal human skin tissues (Fig. 2B). The relative protein expression, according to the Raytest TINA software, was an average of $0.435(\mathrm{SD}=0.130)$ in normal skin and $0.175(\mathrm{SD}=0.0149)$ in diabetic skin. There were significant differences in protein expression between the normal skin and diabetic skin $(\mathrm{P}<0.05)$.

\section{DISCUSSION}

Human skin is easily exposed to oxidative stress caused by ROS accumulation and yet possesses, at the same time, various antioxidant defense mechanisms to protect itself against oxidative stress.

Superoxide dismutase (SOD) is one family of antioxidant enzymes that regulate detoxification of ROS by catalyzing su-

\section{Fig. 1. Immunohistochemical study}

(A) Extracellular superoxide dismutase (EC-SOD) protein was strongly expressed in normal skin tissue (immunochemical stain, $\times 20$ ). The color brown represents a positive stain. (B) EC-SOD was strongly expressed in the entire epidermis and appendages of normal skin tissue (immunochemical stain, $\times 100$ ). (C) EC-SOD was weakly expressed in diabetic skin (immunochemical stain, $\times 40$ ). (D) EC-SOD was weakly expressed in portions of the epidermis of diabetic skin (immunochemical stain, $\times 100$ ).
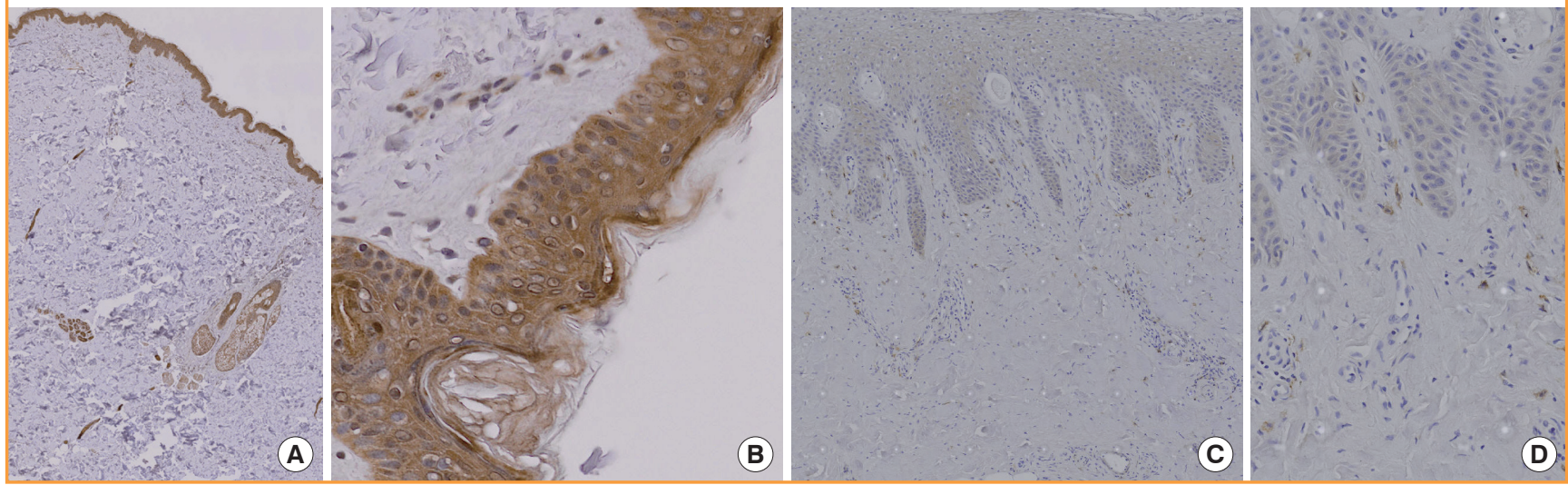

\section{Fig. 2. Western blot analysis and relative protein expression of EC-SOD}

(A) Western blot analysis showed that extracellular superoxide dismutase (EC-SOD) was less expressed in diabetic skin tissues compared with normal human skin tissues. (B) The relative protein expression according to the Raytest TINA software was an average of 0.435 (standard deviation $[S D]=0.130)$ in normal skin and $0.175(S D=0.0149)$ in diabetic skin. There were significant differences in protein expression between normal skin and diabetic skin $(P<0.05)$. N, normal skin tissue; $D$, diabetic skin tissue.

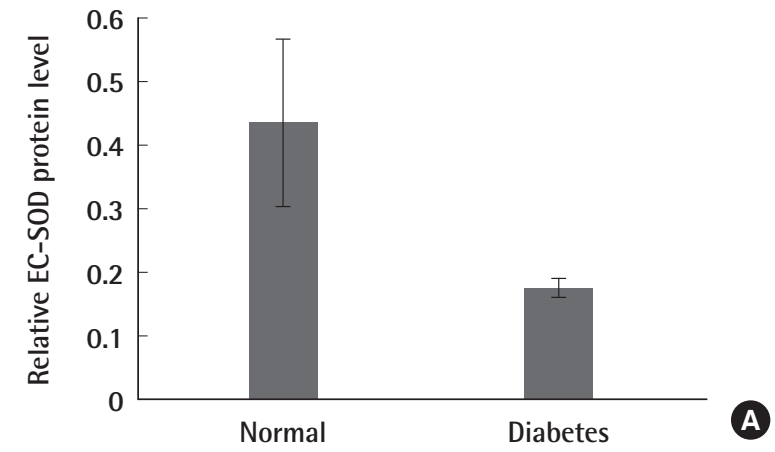


peroxide radicals to hydrogen peroxide and oxygen [9]. At present, three isoforms of SOD, cytoplasmic CuZn-SOD (SOD1), mitochondrial Mn-SOD (SOD2), and extracellular SOD (ECSOD, SOD3), have been identified in mammalians [1]. CuZnSOD and Mn-SOD are thought to act as bulk scavengers of superoxides in the cell $[10,11]$. Mn-SOD has been shown to play a major role in promoting cellular differentiation and tumorgenesis [12]. EC-SOD is an important defense mechanism against superoxide radicals released into the extracellular matrix microenvironment in the skin, and EC-SOD is involved in signal transduction of cell [11].

The human EC-SOD gene is localized to chromosome 4p16.3-q21 [13], and it exists as a homotetramer of molecular weight $135,000 \mathrm{Da}$ with a heparin-binding domain that easily binds to connective tissues [14].

It is well-known that diabetes increases oxidative stress in both animal models and diabetic patients [3]. Oxidative stress is involved in pancreatic beta-cell damage, insulin resistance, and vascular dysfunction. Several reports suggest that the ECSOD gene is one of the candidate genes for diabetes. Adachi et al. [15] reported that plasma levels of EC-SOD were associated with insulin resistance in diabetes. Also, they demonstrated that serum concentrations of EC-SOD were significantly higher in those with diabetes [16]. Kimura et al. [8] reported a positive correlation between serum EC-SOD concentration levels and the severity of diabetic vascular complications, such as nephropathy and retinopathy. In EC-SOD knockout mice, Sentman et al. [17] showed that alloxan-induced pancreatic beta-cell damage was enhanced and recovery from hyperglycemia was delayed. Yamada et al. [18] demonstrated that diabetic patients who had the polymorphism of the EC-SOD gene had an increased risk of ischemic cardiovascular and cerebrovascular diseases.

Proteins are significant targets for oxidative stress. In this study, EC-SOD protein was measured in diabetic skin tissue and normal tissue with western blot and immunohistologic methods. To our knowledge, EC-SOD in diabetic skin tissue and normal tissue has not yet been studied. We used the western blotting for quantitative analysis and found differences in the diabetic skin tissues and normal skin tissues. The relative protein expression calculated with the Raytest TINA software was an average of $0.435(\mathrm{SD}=0.130)$ in normal skin and $0.175(\mathrm{SD}=0.0149)$ in diabetic skin. There were significant differences in protein expression between normal skin and diabetic skin $(\mathrm{P}<0.05)$. The immunohistochemical method revealed that EC-SOD was weakly expressed in diabetic skin.

In conclusion, it was found that diabetic skin tissues express relatively small amounts of EC-SOD protein and may not be protected against oxidative stress. Hence, we believe that EC-
SOD is related to the altered metabolic state of diabetic skin, which elevates ROS production. Further research is needed in order to more precisely determine the mechanisms of EC-SOD.

\section{REFERENCES}

1. Zelko IN, Mariani TJ, Folz RJ. Superoxide dismutase multigene family: a comparison of the CuZn-SOD (SOD1), MnSOD (SOD2), and EC-SOD (SOD3) gene structures, evolution, and expression. Free Radic Biol Med 2002;33:337-49.

2. Bonnefont-Rousselot D, Bastard JP, Jaudon MC, et al. Consequences of the diabetic status on the oxidant/antioxidant balance. Diabetes Metab 2000;26:163-76.

3. West IC. Radicals and oxidative stress in diabetes. Diabet Med 2000;17:171-80.

4. Brownlee M. Biochemistry and molecular cell biology of diabetic complications. Nature 2001;414:813-20.

5. Chance B, Sies H, Boveris A. Hydroperoxide metabolism in mammalian organs. Physiol Rev 1979;59:527-605.

6. Fukai T, Folz RJ, Landmesser U, et al. Extracellular superoxide dismutase and cardiovascular disease. Cardiovasc Res 2002;55:239-49.

7. Stralin P, Karlsson K, Johansson BO, et al. The interstitium of the human arterial wall contains very large amounts of extracellular superoxide dismutase. Arterioscler Thromb Vasc Biol 1995; 15:2032-6.

8. Kimura F, Hasegawa G, Obayashi H, et al. Serum extracellular superoxide dismutase in patients with type 2 diabetes: relationship to the development of micro- and macrovascular complications. Diabetes Care 2003;26:1246-50.

9. McCord JM, Fridovich I. Superoxide dismutase. An enzymic function for erythrocuprein (hemocuprein). J Biol Chem 1969;244:6049-55.

10. Fridovich I. Superoxide radical and superoxide dismutases. Annu Rev Biochem 1995;64:97-112.

11. Oury TD, Day BJ, Crapo JD. Extracellular superoxide dismutase: a regulator of nitric oxide bioavailability. Lab Invest 1996;75:617-36.

12. St Clair DK, Oberley TD, Muse KE, et al. Expression of manganese superoxide dismutase promotes cellular differentiation. Free Radic Biol Med 1994;16:275-82.

13. Hendrickson DJ, Fisher JH, Jones C, et al. Regional localization of human extracellular superoxide dismutase gene to 4pter-q21. Genomics 1990;8:736-8.

14. Marklund SL. Human copper-containing superoxide dismutase of high molecular weight. Proc Natl Acad Sci U S A 1982;79:7634-8.

15. Adachi $T$, Inoue $M$, Hara $H$, et al. Relationship of plasma ex- 
tracellular-superoxide dismutase level with insulin resistance in type 2 diabetic patients. J Endocrinol 2004;181:413-7.

16. Adachi T, Nakamura $M$, Yamada $H$, et al. Quantitative and qualitative changes of extracellular-superoxide dismutase in patients with various diseases. Clin Chim Acta 1994;229: 123-31.

17. Sentman ML, Jonsson LM, Marklund SL. Enhanced allox- an-induced beta-cell damage and delayed recovery from hyperglycemia in mice lacking extracellular-superoxide dismutase. Free Radic Biol Med 1999;27:790-6.

18. Yamada H, Yamada Y, Adachi T, et al. Protective role of extracellular superoxide dismutase in hemodialysis patients. Nephron 2000;84:218-23. 